\title{
Application of Macro Response Monte Carlo method for electron spectrum simulation
}

\author{
L. A. Perles* , A. de Almeida \\ Departamento de Física e Matemática \\ Faculdade de Filosofia, Ciências e Letras de Ribeirão Preto \\ São Paulo University - USP - Brazil
}

\begin{abstract}
During the past years several variance reduction techniques for Monte Carlo electron transport have been developed in order to reduce the electron computation time transport for absorbed dose distribution. We have implemented the Macro Response Monte Carlo (MRMC) method to evaluate the electron spectrum which can be used used as a phase space input for others simulation programs. Such technique uses probability distributions for electron histories previously simulated in spheres (called kugels). These probabilities are used to sample the primary electron final state, as well as the creation secondary electrons and photons. We have compared the MRMC electron spectra simulated in homogeneous phantom against the Geant 4 spectra. The results showed an agreement better than $6 \%$ in the spectra peak energies and that MRMC code is up to 12 time faster than Geant4 simulations.
\end{abstract}

Key words: electron spectrum, variance reduction techniques, Monte Carlo

\section{Introduction}

Currently, modeling electron beam treatment planning system is a challenging task. Several methods have been provided to calculate the tridimentional (3D) dose distributions, from Hogstron analytical approximation [1] to Monte Carlo (MC) simulations. The simulations with MC code, using condensed history, are accurate but usually require several hours to achieve reasonable statistics. To reduce the simulation time several variance reduction techniques have been proposed such as Response History Monte Carlo (RHMC) [2], Macro Monte Carlo (MMC) 34, Phase-space evolution Monte Carlo (Evolution) [5], Macro Response Monte Carlo (MRMC) [6] and Voxel Monte Carlo (VMC) [7].

\footnotetext{
* Corresponding author.

Email address: perles@gmail.com (L. A. Perles).
}

The RHMC, MMC, Evolution and MRMC codes use probability distributions generated from electron histories previously simulated in a volume element, to transport the primary electron and create secondary ones. The Evolution generates its phase space in cubic voxels, the RHMC generates in hemispheres and MMC and MRMC generate in spheres (kugels). The VMC code relies on some simplifications of the models for electron transportation and history repetition.

The cited codes have shown an accuracy up to $5 \%$ in the depth dose, but only RHMC, MMC and VMC have achieved a real speed gain, when compared with others MC codes. Basically, the use of voxels instead of kugels is the limitation of the Evolution code because it is difficult to overcome the symmetry problem. The MRMC limitation, mentioned by the author, was related to the small radii of the kugels used.

We have implemented the MRMC method with 
larger radii as well more radii choice for electron transportation. Such implementation can be used for generating electron spectra, since it does not have dose deposition algorithm implemented.

\section{Methods}

\section{1. $M R M C$ implementation}

The MRMC simulation is divided into two parts called local and global calculations. In the first one we have simulated the kugels database for water, soft tissue and compact bone. In the second part is the electron interaction simulation in the materials and phantom geometries selected.

\subsubsection{Local calculation}

The local calculation consisted in the simulation of electron histories starting from kugel's center with initial direction aligned to the $\mathrm{Z}$ axis, figure 1. The particles' state leaving the kugel is tailed in probability distribution histograms.

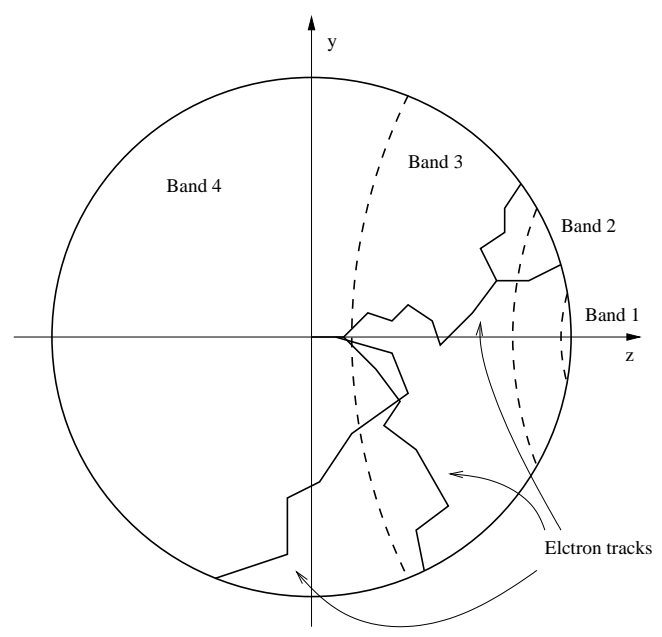

Figure 1. Illustration of kugel bands with some electron tracks and its output band.

The kugel is divided into 4 bands along the $\mathrm{Z}$ axis, where the projections over such axis are: 1.0 to 0.98 (band 1), 0.98 to 0.8 (band 2), 0.8 to 0.4 (band 3 ) and 0.4 to -1.0 (band 4 ). Each of these bands has a complete set of probabilities distribution histograms comprising one for the output positions, two for directions and one for energies. These quantities have been divided into three groups: primary electrons, secondary electrons and photons. Also, a histogram had been generated to store the average number of escaping secondary electrons from the kugel geometry and another one for photons escaping.

Each material has a complete set of several kugel radii and energies. The radii chosen were: 0.025 , $0.050,0.100,0.250,0.500$ and $1.000 \mathrm{~cm}$, where only the first three were tested in the original work. We have chose 19 energies stepping from $31 \mathrm{MeV}$ down to $178 \mathrm{keV}$ with an energy spacing around $25 \%$, as suggested by Svatos [6].

We have used the Geant4 version $8.0[8]$ to simulate the kugels database. Such code uses a condensed history for electron transportation, while in the original implementation of the MRMC the author have used a Single Scattering MC code. Each kugel has been simulated with $5 \times 10^{5}$ histories, using $990 \mathrm{eV}$ as electron cutoff and $10 \mu \mathrm{m}$ as rangecut for water and soft tissue and $20 \mu \mathrm{m}$ for compact bone. Due to long elapsed time, these simulations took place in a small cluster.

The database was stored in ROOT [10] file format, which properly store kugel data for global calculations.

\subsubsection{Global calculation}

The global calculation is the electron transport through the phantom geometry using kugels to sample the primary electron final state, as well the secondary electrons and photons production. An activity diagram, in Unified Modeling Language (UML), is presented in the figure 2, First, the system tries to find an energy through a linear-logarithmic statistical interpolation, expression 11, where $E$ is the energy of electron being transported, $E_{+}$and $E_{-}$are the nearest energies available in the database and $\chi$ is an uniform random number in $\left[0,1\left[\right.\right.$. The $E_{+}$is chosen when the expression 1 is true, otherwise $E_{-}$ will be chosen.

$\chi>\frac{\log E_{+}-\log E}{\log E_{+}-\log E_{-}}$

After an energy is chosen, the system searches for a radius that does not cross any boundary. If there is a kugel, the MRMC code system uses it to sample the particles final state, including the secondaries and photons production. If there is no kugel, the system transports the current electron using an straight line approximation and corrects its energy using a Continuous Slowing Down Approximation (CSDA). The secondaries are transported as the primary ones and the photons are transported until they leave the geometry, since interaction models are no implemented. 


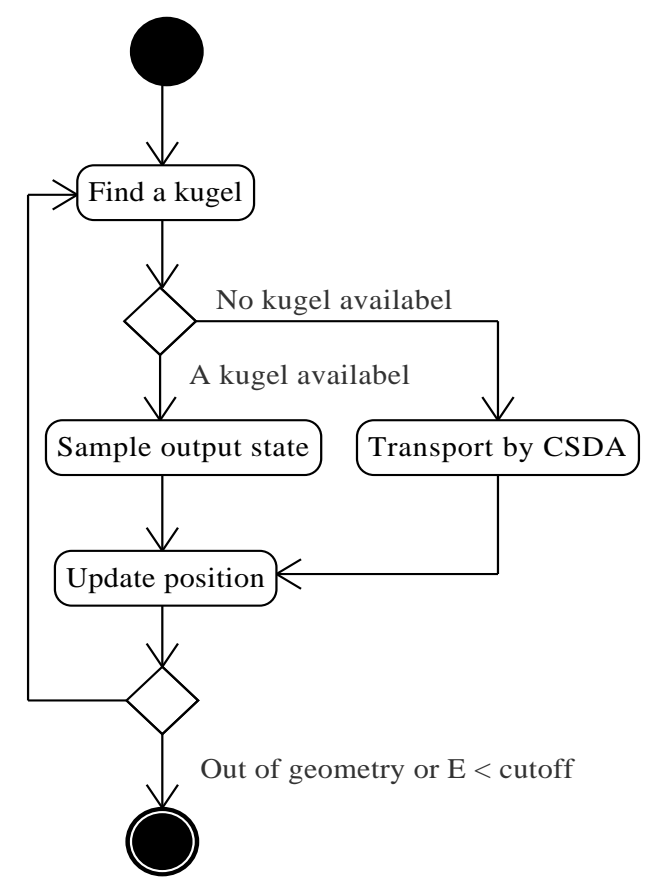

Figure 2. UML Activity diagram showing the electron logic transportation.

The MRMC was written in object oriented style using $\mathrm{C}++$ language, with the ROOT library to sample the histograms and for the particle transportation in the geometry.

\subsection{Benchmarks}

The tests consisted in an electron pencil beam incident perpendicularly in a homogeneous cylindrical phantom. The electron beam starts at $0.025 \mathrm{~cm}$, inside the phantom, in order to avoid the straight line approximation in the beginning of event. The simulation parameters for the MRMC were set to $5 \mathrm{keV}$ for both, the cutoff and secondaries threshold. The Geant4 parameters were set for $1 \mathrm{keV}$ of cutoff and $0.1 \mathrm{~mm}$ of rangecut.

We compared the forward and lateral electron spectra scatter against the Geant 4 simulations. The energies chosen for these tests were: 10.0, 8.0, 7.5, 5.0, 3.0 and $1.0 \mathrm{MeV}$.

\section{Results}

\subsection{Electron spectra}

The forward and lateral spectra simulated by MRMC for water and soft tissue have shown an agreement better than $2 \%$ in the peak energies for the incident energies, above $3 \mathrm{MeV}$, and up to $5 \%$ for incident energies below $3 \mathrm{MeV}$, figures 3 to 5 . For compact bone the results showed an agreement better than $6 \%$ for the incident energies above $5 \mathrm{MeV}$ and better than $2 \%$ for the incident energies below $5 \mathrm{MeV}$, figures 6 and 7 . Such deviations may be due to uncertainties in the local simulations done with Geant4, straight line approximation for boundary cross and uncertainties in total stopping power tables [11.

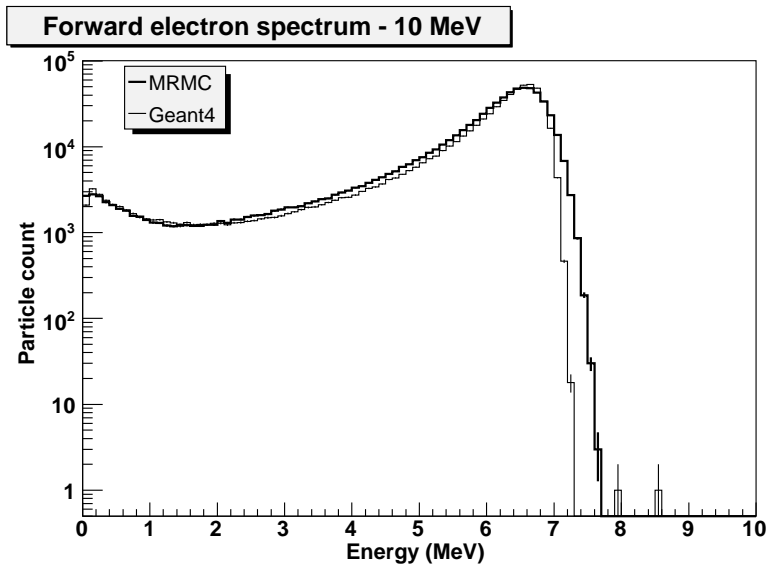

Figure 3. Forward electron spectrum for incident energy of $10 \mathrm{MeV}$ in water phantom.

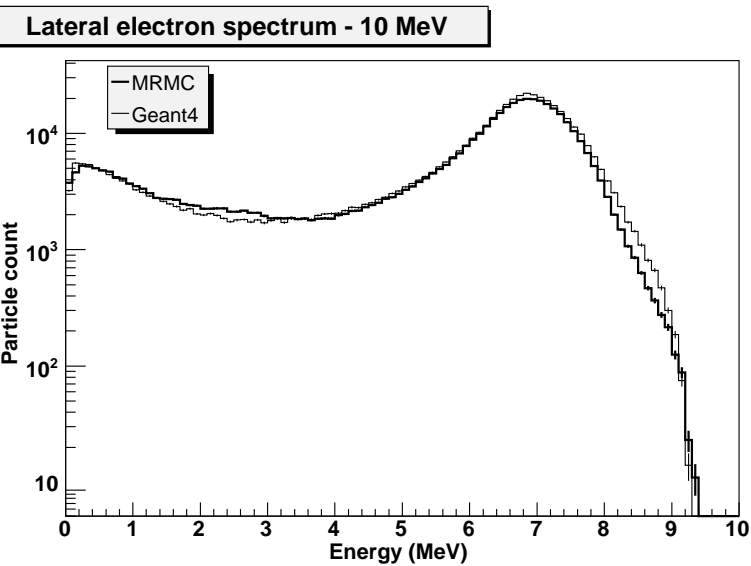

Figure 4. Lateral electron spectrum for incident energy of $10 \mathrm{MeV}$ in water phantom.

\subsection{Simulation speed}

The MRMC simulation speed was around three times faster for lowest energy and up to 12 times faster for $10 \mathrm{MeV}$. Simulations for low energies are 


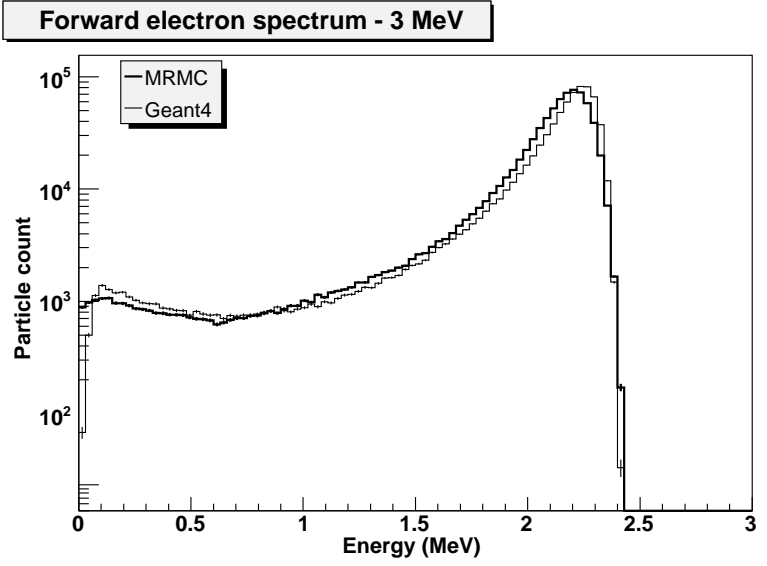

Figure 5. Forward electron spectrum for incident energy of $3 \mathrm{MeV}$ in soft tissue phantom.

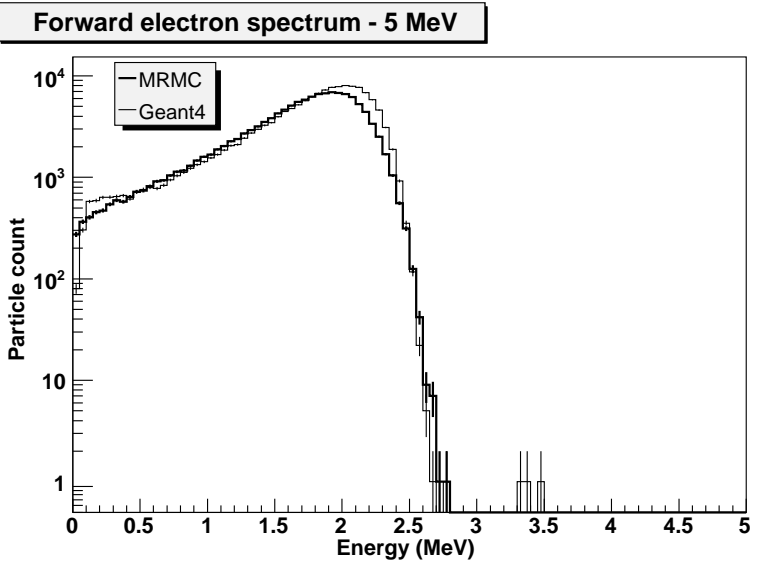

Figure 6. Forward electron spectrum for incident energy of $5 \mathrm{MeV}$ in compact bone phantom.

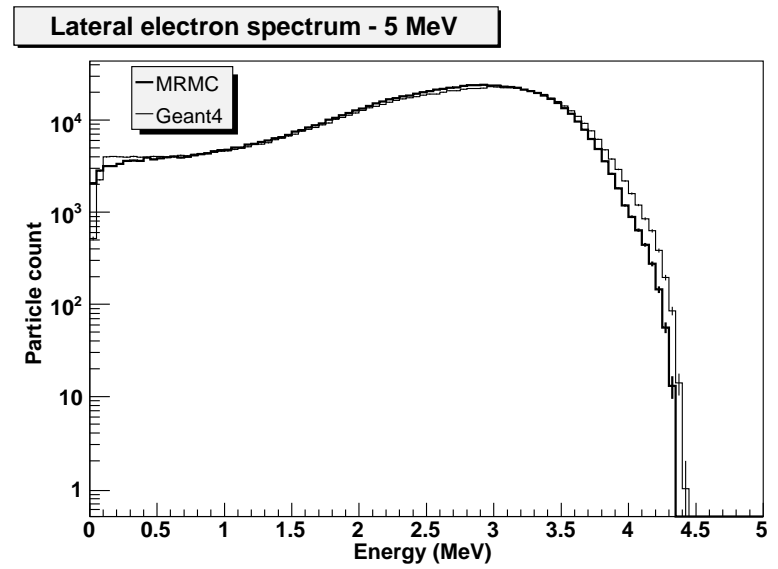

Figure 7. Lateral electron spectrum for incident energy of $5 \mathrm{MeV}$ in compact bone phantom.

slower because the database for such energies have only smaller kugels radii available.

\section{Conclusions}

The simulated spectra from our implementation of MRMC code have shown good agreement and the speed was up to 12 time faster, when compared to Geant4 for energies from 1 to $10 \mathrm{MeV}$.

The authors would like to thank to prof. Dr. Antonio Carlos Roque da Silva Filho and Dr. Rodrigo Freire Oliveira to allow the access to cluster for our calculations. Also, we would like to thank to $\mathrm{CNPq}$ for the financial support.

\section{References}

[1] K. Hogstrom, M. Mills, P. Almond, Electron beam dose calculations, Phys. Med. and Biol. 26 (3) (1981) 445459.

[2] C. Ballinger, J. Rathkopf, W. Martin, The Response History Monte Carlo method for electron transport, Nucl. Sci. Eng. 112 (1992) 283-95.

[3] H. Neuenschwander, E. Born, A Macro Monte Carlo method for electron beam dose calculations, Phys. Med. Biol. 37 (1) (1992) 107-125.

[4] H. Neuenschwander, T. Mackie, P. Reckwerdt, MMC-a high-performance Monte Carlo code for electron beam treatment planning, Phys. Med. Biol. 40 (1995) 543-574.

[5] D. Scora, B. Faddegon, Monte carlo based phase-space evolution for electron dose calculation, Med. Phys. 24 (2) (1997) $177-187$.

[6] M. M. Svatos, The Macro Response Monte Carlo method for electron transport, $\mathrm{Ph}$.D. thesis, Lawrence Livermore National Laboratory (1998).

[7] I. Kawrakow, M. Fippel, K. Friedrich, 3D electron beam dose calculation using a Voxel based Monte Carlo algorithm (VMC), Med. Phys. (23) (1996) 445-457.

[8] S. Agostinelli, et al., Geant4-a simulation toolkit, Nucl. Instrum. and Meth. A 506 (2003) 250-303.

[9] J. Allison, et al., Geant4 developments and applications, Nuclear Science, IEEE Transactions on 53 (1) (2006) 270-278.

[10] R. Brun, F. Rademakers, P. Canal, I. Antcheva, D. Buskulic, ROOT User's Guide, CERN / FNAL / LAPP, version 5.08 (Dec. 2005).

[11] INTERNATIONAL COMMISSION ON RADIATION UNITS AND MEASUREMENTS, ICRU Report 37 - Stopping powers for electrons and positrons, Tech. rep., International Comission on Radiation Units and Measurements, Washington, DC (1984). 


\section{Espectro frontal dos elétrons - $5 \mathrm{MeV}$}

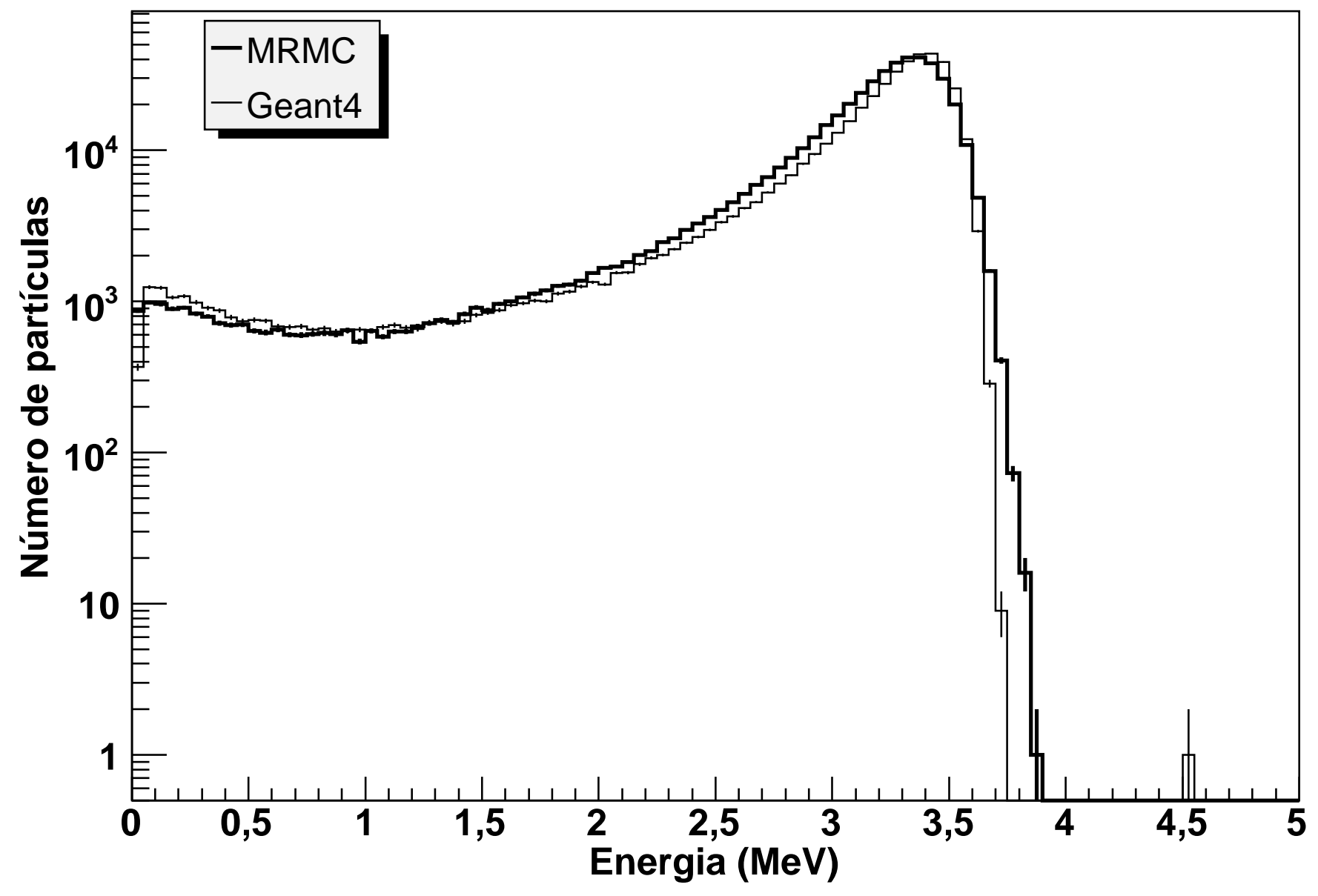




\section{Espectro lateral dos elétrons - $5 \mathrm{MeV}$}

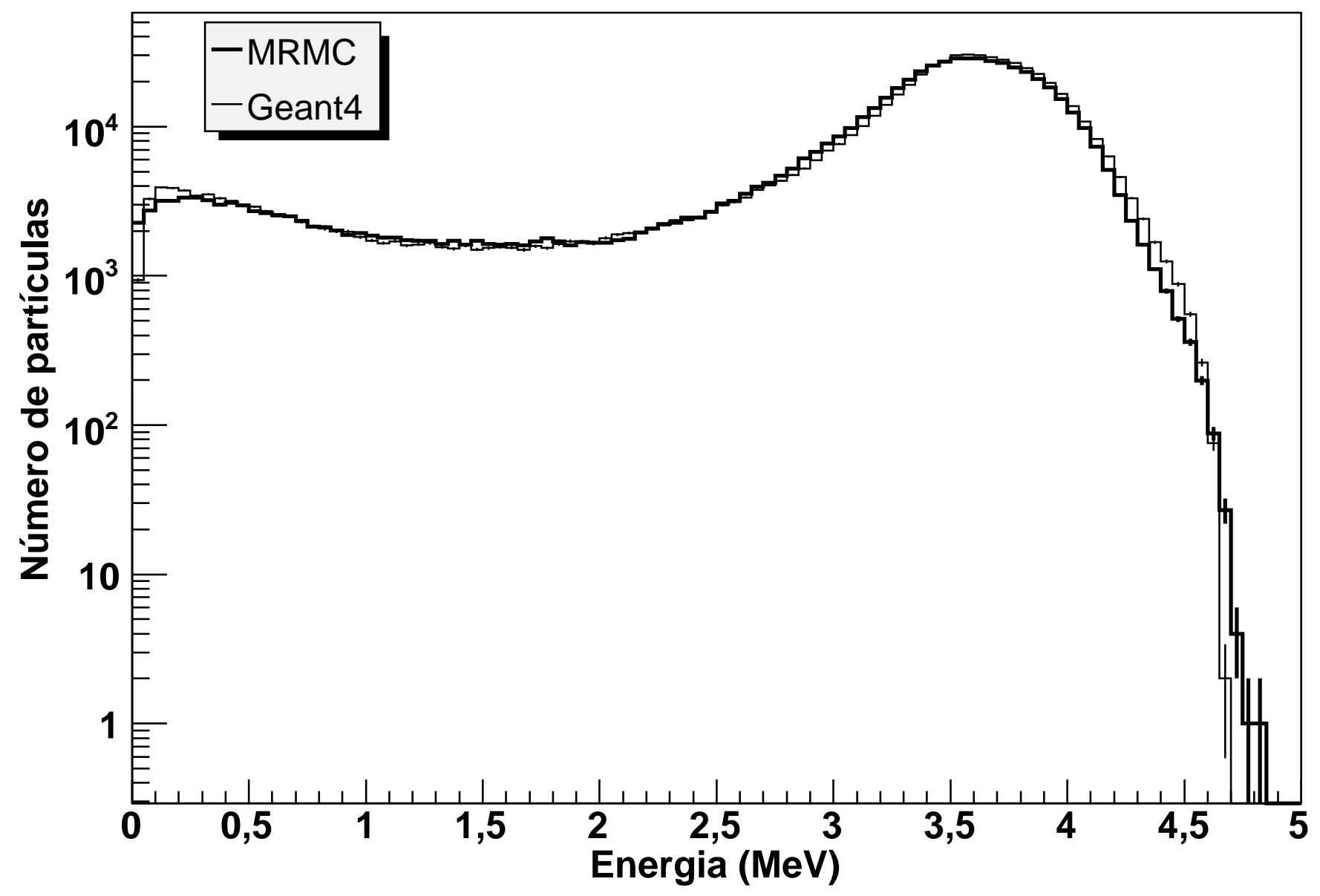

